\title{
Dental anomalies in fetal alcohol syndrome. A systematic review
}

\author{
Rodrigo Soares de Andrade ${ }^{1 *}$, Ricardo Martinez Pedraza ${ }^{2}$, Daniella Reis Barbosa Martelli1 ${ }^{3}$, Jesus Alfonso Aguirre Quiñones ${ }^{4}$ and Hercílio \\ Martelli Júnior ${ }^{5}$ \\ ${ }^{1}$ Centro Universitário de Patos de Minas (UNIPAM) (Department of Oral Diagnosis), Patos de Minas (MG), Brazil \\ ${ }^{2}$ Facultad de Odontologia, Universidad Autonoma de Nuevo Leon, Monterrey, Nuevo Leon, Mexico \\ ${ }^{3}$ Dentistry School, Universidade Estadual de Montes Claros (UNIMONTES), Montes Claros (MG), Brazil \\ ${ }^{4}$ Facultad de Odontologia, Unidad Torreon, Universidad Autonoma de Coahuila, Torreon, Coahuila, Mexico \\ ${ }^{5}$ Department of Oral Diagnosis, Faculdade de Odontologia de Piracicaba, Universidade Estadual de Campinas, Piracicaba (SP), and Full Professor in Dentistry \\ School, Universidade Estadual de Montes Claros, Montes Claros, Brazil
}

\begin{abstract}
Background: Fetal alcohol syndrome (FAS) is the set of signs and symptoms presented by the fetus resulting from maternal alcohol ingestion during pregnancy and preconception periods, such as growth deficit, facial features alterations and delayed neuropsychomotor development, as well as dental anomalies.

Objective: The aim of this systematic review was to investigate the different dental anomalies associated with FAS.

Methods: A Medline search for "fetal alcohol syndrome and dental anomalies" was performed. Further research was conducted for "fetal alcohol syndrome" and its individual oral manifestation. The inclusion criteria were: human clinical studies without a minimum of stipulated patients. Nine studies fulfilled the inclusion criteria and were analyzed accordingly to PRISMA protocol for systematic reviews.

Results: A total of 211 patients with FAS were included in the analyzed studies. The nine studies analyzed included case reports, case series and a review on the subject. Cardiac, respiratory, renal and cognitive defects were the main systemic manifestations, while the main dental abnormalities found were malocclusions, misplaced teeth in the dental arch, tooth agenesis and supernumerary teeth.

Conclusion: Although Oral and systemic manifestations are always found in patients with FAS, such common dental anomalies are poorly documented and insufficiently described, and given the importance of such aspects for the patient's health, we suggest that more studies on this subject are necessary to describe the possible consequences from those anomalies.
\end{abstract}

\section{Introduction}

Fetal alcohol syndrome (FAS) refers to a pattern of congenital malformations seen in children born to women who consumed large amounts of alcohol during pregnancy [1]. FAS hallmarks have been catalogued, quantified, and refined over the years and many investigators have established that most consequences of maternal alcohol consumption during pregnancy are pre and post-natal growth deficiency as well as brain and craniofacial abnormalities [1-8]. FAS was first described in 1973 in a study reporting a variety of growth and developmental abnormalities in 11 children whose mothers drank heavily during their pregnancies [9]. The symptoms can be found from in-utero up to early childhood. There is a wide variety of developmental defects that result from alcohol exposure, including brain abnormalities, central nervous system dysfunctions, and growth deficiencies of developing organs and body systems [5]. These adverse effects on the developing fetus are collectively known as fetal alcohol spectrum disorders (FASD). FAS cause learning, emotion, cognition and motor performance dysfunctions, while also possibly leading to behavioral as well as social issues [10].

Moreover, Orofacial abnormalities are extremely common and an important part of the FAS diagnosis [5,6]. Growth deficits, facial characteristics alterations and neuropsychomotor development delay are all observed [11] and systemic manifestations include low birth weight [8], low weight gain [7], microcephaly (small head) [7], difficulty in learning, language, memory and attention [5], low Intelligence quotient (IQ) [6], changes in vision and hearing [9], difficulties of socialization [10], behavioural disorders [12], delayed cognitive development [11], kidney diseases [8], neurological changes such as seizures, kidney, bone, and congenital heart disease [9]. New-born infants with FAS may be irritable, exhibit muscular hypotonia, severe tremors, and withdrawal symptoms. Mild mental retardation is the most common and serious deficit, while a variety of other anomalies affecting the nervous system, hearing, and organs may accompany FAS [12]. The signs and symptoms are diverse since the passage of ethanol

*Correspondence to: Rodrigo Soares de Andrade, Centro Universitário de Patos de Minas (UNIPAM) (Department of Oral Diagnosis), Av. Marabá, 831 - Alto dos Caicaras, Patos de Minas - MG, 38703-236, Brazil, Tel: (+55 19) 99966-5488, E-mail: rodrigosoares002@hotmail.com

Key words: fetal alcohol syndrome, oral health, anodontia

Received: January 20, 2020; Accepted: February 04, 2020; Published: February 13, 2020 
through the placenta and the degree of metabolization of ethanol by the maternal liver are both variable [8]. The most common orofacial changes are small eyelid fissures [11], flat facies [12], maxillary hypoplasia [6], short nose [6], long and hypoplastic nasal filter [9], and thin upper lip [10]. The unique facial appearance of FAS patients is the result of changes in 4 areas: short palpebral fissures, flat nasal bridge with an upturned nasal tip, hypoplastic philtrum with a thin upper vermillion border, and a flat midface [11]. Other facial anomalies include micrognathia [13], occasional cleft lip and/or palate and small teeth with defective enamel [3]. Therefore, the aim of this systematic review was to investigate the different dental anomalies described and associated with FAS.

\section{Methods}

The present review was carried out in accordance with the PRISMA protocol for systematic reviews [14].

Search strategies: An electronic search in PubMed (MEDLINE), Scopus, Embase, Google Scholar and The Cochrane Library was conducted by two independent reviewers until December 2018, when we researched the following terms following the PICO search strategy (http://askmedline.nlm.nih.gov/ask/pico.php): 'Fetal alcohol syndrome and dental anomalies' [title/abstract] OR 'Fetal alcohol syndrome and dental alterations" [title/abstract] OR 'Fetal alcohol syndrome and dental manifestation' [title/abstract] OR 'Fetal alcohol syndrome and oral manifestation' [title/abstract], OR “Fetal alcohol syndrome and oral findings" [title/abstract] including a literature search strategy.

Inclusion and exclusion criteria: Selection of papers through the inclusion and exclusion of criteria, data extraction, and quality assessment. Meta-analysis was not possible since selected studies did not observe the same variables, methods, participants, and outcomes, which prevented comparisons. A manual search by reading the references of the selected studies was also performed.

Study selection: Prospective or retrospective cohort studies, case series and case reports describing clinical and/or laboratorial characteristics of patients with dental anomalies in FAS were included. The titles and abstracts of all reports in the electronic searches were each read by two investigators separately. The studies that fulfilled the inclusion criteria and those that did not present sufficient information in the title or in the abstract, were fully assessed. A cross-check assessment of the articles was performed by another author to guarantee that the proper studies were selected according to the inclusion and exclusion criteria.

Data extraction: We independently extracted study data through specific extraction forms using Microsoft Excel software. For the study selected, the following information was extracted (when available): year and country of publication, article type, number of cases, sex, if consanguinity existed in the patients (which could lead to a search), systemic manifestations and dental alterations described by the authors.

Analysis: All the patients' clinical information and diagnostic criteria were evaluated for data descriptive cross-referencing, considering the information given by the authors, wherein the main dental alterations were observed in FAS-affected patients.

\section{Results}

The searches returned a total of 74 records. After the duplicates were removed, 35 records remained (Figure 1). All 74 articles were selected through the titles and abstracts and 29 records were excluded

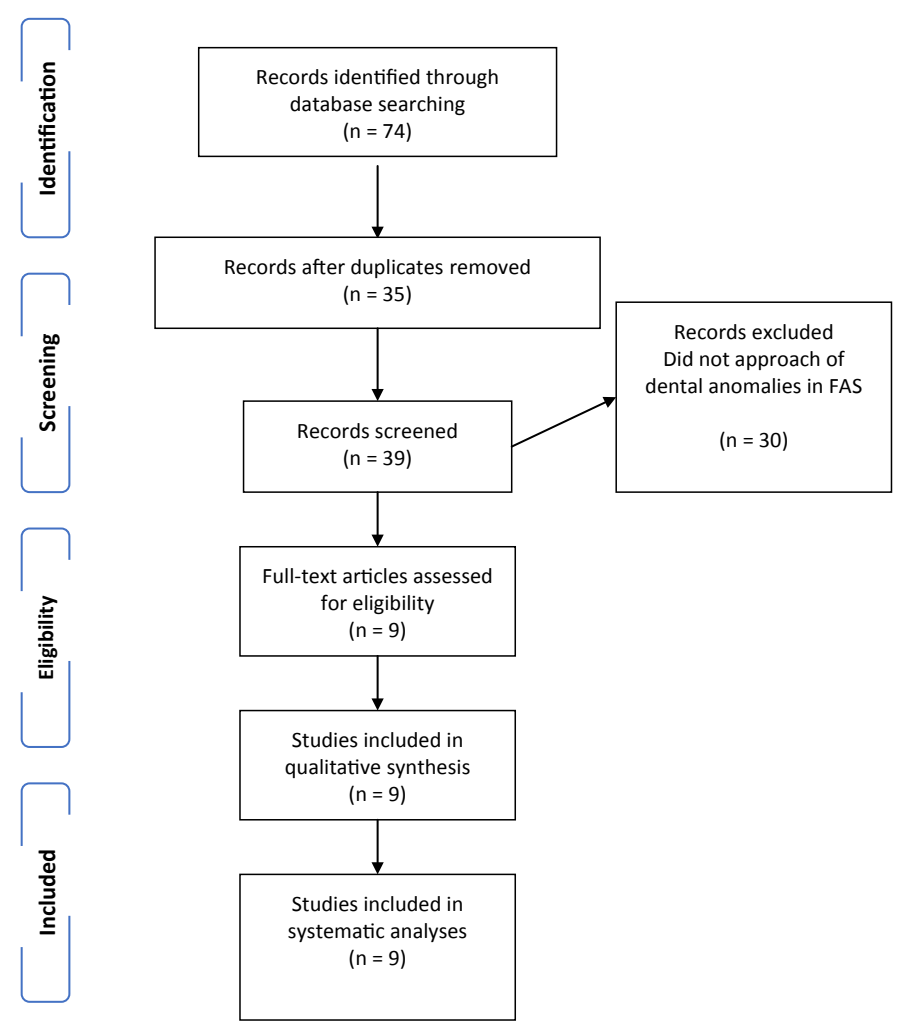

Figure 1. Flowchart of process of systematic literature search, using PRISMA flow.

based on the exclusion criteria. Of the excluded records, 65 did not focus on the presence of dental anomalies in the FAS, of which 4 were reports of animal tests, 47 reported facial changes without anomalies mentions and 11 were deemed irrelevant to the topic, resulting in 9 papers that met the search criteria, classified as follows: 3 case series, 3 case reports, 2 original research article and 1 letter to the editor [15-24]. All cited and selected papers had in their methodology and in their discussion the focus on showing oral, facial and systemic alterations in patients with FAS (Table 1). All articles followed the 1966 IOM (Institute of Medicine Diagnostic) criteria, even those that were published before that date were reviewed. The paper by Streissguth et al., [4,5] was the first article describing malocclusions and changes in dental position found in patients with FAS [16] while also describing other facial and systemic alterations of patients with FAS. However, Chuch et al., was the first one that specifically described the number and shape of dental anomalies in serial cases of 22 patients and cited the presence of agenesis, twisting tooth and diastema. 8 of the papers cited the presence of oral clefts in the reported cases [15-23]. In the paper by Andrade [24], it was clearly shown the presence of dental alterations and abnormalities and made their correlation with systemic renal alterations, which are derived from the same genetic defect of numeral dental alterations.

\section{Discussion}

FAS is characterized by a pattern of congenital anomalies that include pre and/or postnatal growth retardation, central nervous system involvement with neurologic abnormalities, developmental delay or intellectual impairment, and various facial birth defects [1-5,8,15$18,22]$. It has been reported that during the prepubertal age, FAS patients have a characteristic emaciated appearance, which may not remain a feature in adolescence, at least in females [10-12,23]. Diagnostic criteria 
Table 1. Papers used in the systematic review of fetal alcohol syndrome, showing the main oral and systemic manifestations of the syndrome.

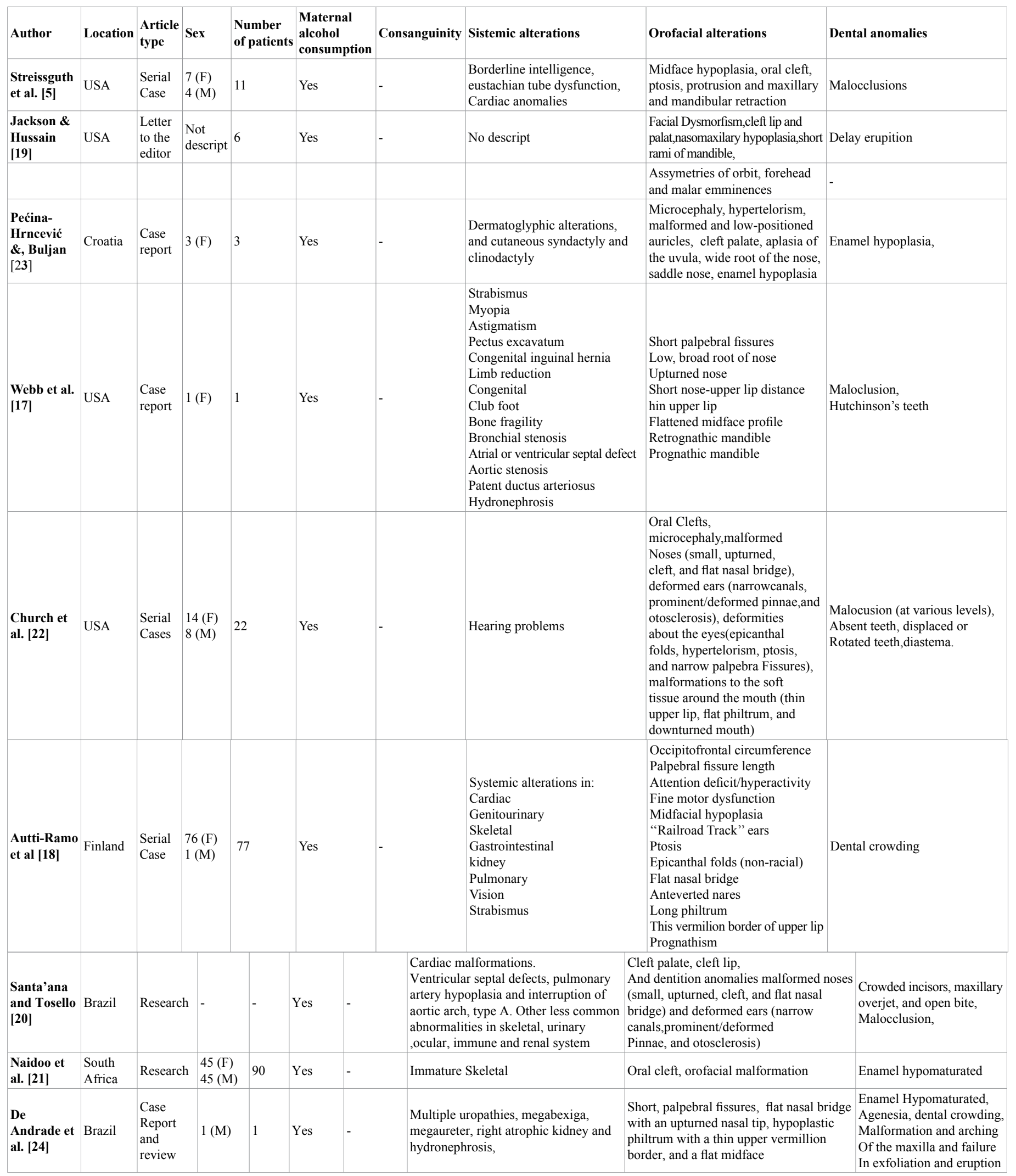

Legend: $\mathbf{U S A}=$ United States of America,

$\mathbf{F}=$ female, $\mathbf{M}=$ male 
for FAS developed by IOM simplified the identification of classic FAS cases. When the phenotype is less than classic or atypical, confirming the diagnosis becomes difficult, if not impossible [17,18,20]. Early diagnosis is essential for appropriate intervention on children affected by prenatal alcohol exposure and can reduce their risk of facing social difficulties later in life as well as employment issues due to behavioural and learning difficulties or even lawbreaking occurrences as a result of impulsive behaviour and lack of inhibition [15-24]. In the papers presented, FAS diagnosis was made through the association of facial and systemic alterations, and the presence of several dental anomalies, such as agenesis, malocclusions, diastemas, twisting tooth and dental crowds [15-24]. Naidoo et al observed a reduced age of dental maturation in 90 patients with FAS compared to a normal patient showing a slight alteration in development characterized by delayed enamel maturation [21]. Andrade et al., [24] observed its applicability in the case report in which boy shows enamel hypomaturation and agenesis of several teeth due to an acute kidney disease, which is also described in the work of Autti-Ramo et al. (2005) and Sann'tanna et al. (2006).

It has been documented that alcohol consumption during pregnancy can be a cause of orofacial cleft [25]. Munger et al., [26] reported that maternal alcohol consumption during pregnancy was associated with an increased risk of cleft lip with or without cleft palate. Furthermore, the offspring of alcohol-consuming mothers showed a higher chance of developing cleft lip and palate were higher compared to women who did not consume alcohol during pregnancy [26]. The number of distinct teratogenic mechanisms works simultaneously along different biochemical pathways and at different physical locations in the developing embryo [27]. The ways in which this alcohol-induced mechanism damage the fetus depends on several variables, such as time, frequency, and the amount of maternal alcohol consumption during pregnancy; the state of health and habits of the mother as well as the genetic constitution of the mother and the fetus. These teratogenic effects directly affect dental anomalies presence in FAS [28]. Dental and maxillofacial alterations such as orofacial fissures are due to the teratogenic alcohol effect on the fetus, which as described in Sant'anna's et al. (2006), causes alteration in the MSX1 and MSX2 gene, both participating in the maxillary process's fusion odontogenic formation, a hypothesis reinforced by Naidoo et al., [13,21]. The most frequently occurring anomalies were crowded incisors, maxillary overjet, and open bite [16-18,20,21]. Levels of plaque and gingival bleeding were high, which was not an unexpected finding since these children come from backgrounds in which oral hygiene is not a high priority [19].

\section{Conclusion}

In this systematic review, where the papers published in the main research platforms were scanned, it is observed that there are few studies that explain and show the direct relationship between oral manifestations and SAF, evidencing the various dental anomalies that these patients present such as agenesis, malocclusions, diastemas, twisting tooth and dental crowds. More studies are needed to explore these dental alterations, since they cause aesthetic and functional problems in these patients who already have several isthmian alterations, thus making the effects of SAF in these patients more discreet.

\section{Sources of funding}

None

\section{Conflict of interest}

None

\section{Prospero register}

\section{CRD42019133863}

\section{Acknowledgment}

The Minas Gerais State Research Foundation-FAPEMIG, Minas Gerais, Brazil and the National Council for Scientific and Technological Development - CNPq, Brazil.

\section{Authors' contribution}

RSA designed and wrote the manuscript, RMP and JAQ reviewed the manuscript, DRBM assisted in the search methodology, HMJ oriented and reviewed the manuscript.

\section{References}

1. Hanson JW, Jones KL, Smith DW (1976) Fetal alcohol syndrome. Experience with 41 patients. JAMA 235:1458-1460. [Crossref]

2. Chernoff GF (1997) The foetal alcohol syndrome in mice: an animal model. Teratology 15: 223-239. [Crossref]

3. Clarren SK, Smith D W (1978) The foetal alcohol syndrome. N Engl J Med 298: 1063 1067. [Crossref]

4. Clarren SK, Alvord EC, Sumi SM, Streissguth AP, Smith DW (1978) Brain malformations related to prenatal exposure to ethanol. J Pediatr 92: 64-67. [Crossref]

5. Streissguth AP, Landesman-Dwyer S, Martin JC (1985) Teratogenic effects of alcohol in humans and laboratory animals. Science 209: 353-361. [Crossref]

6. Sulik KK, Johnston MC, Daft PA, Russel WE, Dehart DB (1986) Fetal alcoho syndrome and DiGeorge anomaly: critical alcohol exposur e periods for craniofacial malformations as illustrated in an animal model. Am J Med Genet Suppl 2: 97-112. [Crossref]

7. Coles CD (1994) Critical periods for prenatal alcohol exposure. Alcohol Health Res World 18: 22-29. [Crossref]

8. Weston WM, Greene RM, Uberti M, Pisano MM (1994) Ethanol effects on craniofacial growth and development: implications for study of the foetal alcohol syndrome. Alcohol Clin Exp Res 18: 177-182. [Crossref]

9. Jones KL, Smith DW (1973) Recognition of fetal alcohol syndrome in early infancy Lancet 11: 999-1001. [Crossref]

10. Hoyme HE, May PA, Kalberg WO, Kodituwakku P, Gossage JP, et al. (2005) A practical clinical approach to diagnosis of fetal alcohol spectrum disorders: Clarification of the 1996 Institute of Medicine criteria. Pediatrics 115: 39-47. [Crossref]

11. SantAnna LB, Tosello DO, Pasetto S (2005) Effects of maternal etanol intake on immunoexpression of epidermal growth factor in developing rat mandibular molar. Arch Oral Biol 50: 625-634.

12. Jimenez-Farfa D, Guevara J, Zenteno E, Malagon H, Hernandez- Guerrero JC (2005) EGF-R and erbB-2 in murine tooth development after ethanol exposure. Birth Defects Res A Clin Mol Teratol 73: 65-71. [Crossref]

13. Naidoo S (2004) A foetal alcohol syndrome and the role of the oral health care worker SADJ 59: 158-161. [Crossref]

14. Moher D, Liberati A, Tetzlaff J, Altman DG (2009) The PRISMA Group. Preferred reporting items for systematic reviews and meta-analyses: The PRISMA statement. $J$ Clin Epidemiol 62:1006-1012. [Crossref]

15. Caputo C, Wood E, Jabbour L (2016) Impact of fetal alcohol exposure on body systems: A systematic review. Birth Defects Res C Embryo Today 108: 174-180. [Crossref]

16. Itthagarun A, Nair RG, Epstein JB, King NM (2007) Fetal alcohol syndrome: case report and review of the literature.Oral Surg Oral Med Oral Pathol Oral Radiol Endod 103: 20-25. [Crossref]

17. Webb S, Hochberg MS, Sher MR (1988) Fetal alcohol syndrome: report of case. $J$ Am Dent Assoc 116: 196 -198. [Crossref]

18. Autti-Rämö I, Fagerlund A, Ervalahti N, Loimu L, Korkman M, et al. (2006) Fetal alcohol spectrum disorders in Finland: clinical delineation of 77 older children and adolescents.Am J Med Genet A 140: 137-144. [Crossref] 
19. Jackson IT, Hussain K (1990) Craniofacial and oral manifestations of fetal alcohol syndrome. Plast Reconstr Surg 85: 505-512. [Crossref]

20. Sant'Anna LB, Tosello DO (2006) Fetal alcohol syndrome and developing craniofacia and dental structures. Orthod Craniofac Res 9: 172-185. [Crossref]

21. Naidoo S, Norval G, Swanevelder S, Lombard C (2006) Foetal alcohol syndrome: a dental and skeletal age analysis of patients and controls.Eur J Orthod 28: 247-253. [Crossref]

22. Church MW, Eldis F, Blakley BW, Bawle EV (1997) Hearing, language, speech, vestibular, and dentofacial disorders in fetal alcohol syndrome. Alcohol Clin Exp Res 21: 227-233. [Crossref]

23. Pećina-Hrncević A, Buljan L (1991) Fetal alcohol syndrome--case report. Acta Stomatol Croat 25: 253-258. [Crossref]
24. de Andrade RS, Ferreira SB, Morais Soares CMB, Canhestro MR, Coletta RD, et al. (2018) Oligodontia in a Pediatric Patient with Fetal Alcohol Syndrome. J Clin Tri Cas Rep 1: 104-108.

25. Denny L, Coles S, Blitz R (2017) Fetal Alcohol Syndrome and Fetal Alcohol Spectrum Disorders. Am Fam Physician 96: 515-522. [Crossref]

26. Munger RG, Romitti PA, Daack-Hirsch S, Burns TL, Murray JC, et al. (1996) Materna alcohol use and risk of orofacial cleft birthdefects. Teratology 54: 27-33. [Crossref]

27. Smith SM, Garic A, Flentke GR, Berres ME (2014) Neural crest development in fetal alcohol syndrome. Birth Defects Res C Embryo Today 102: 210-220. [Crossref]

28. Del Campo M, Jones KL (2017) A review of the physical features of the fetal alcohol spectrum disorders. Eur J Med Genet 60: 55-64. [Crossref]

Copyright: $(2020$ Soares de Andrade R. This is an open-access article distributed under the terms of the Creative Commons Attribution License, which permits unrestricted use, distribution, and reproduction in any medium, provided the original author and source are credited. 